\title{
STOCHASTIC OPTIMISATION OF THE INITIAL PRESTRESS FOR A TENSILE MEMBRANE STRUCTURE SUBJECTED TO UNCERTAIN WIND FORCES
}

\author{
Subhrajit Dutta ${ }^{1}$, Siddhartha Ghosh $^{2}$, and Mandar M. Inamdar ${ }^{3}$ \\ ${ }^{1}$ Doctoral candidate \\ Department of Civil Engineering \\ Indian Institute of Technology Bombay \\ Mumbai 400076, India \\ e-mail: subhrajit@civil.iitb.ac.in \\ ${ }^{2}$ Associate Professor \\ Department of Civil Engineering \\ Indian Institute of Technology Bombay \\ Mumbai 400076, India \\ e-mail: sghosh@civil.iitb.ac.in \\ ${ }^{3}$ Associate Professor \\ Department of Civil Engineering \\ Indian Institute of Technology Bombay \\ Mumbai 400076, India \\ e-mail: minamdar@civil.iitb.ac.in
}

Keywords: tensile membrane; reliability-based design optimisation; optimisation under uncertainty; particle swarm optimisation; dynamic relaxation; Latin hypercube sampling.

\begin{abstract}
Tensile membrane structures are lightweight flexible structures that need to remain in equilibrium in the presence of heavy wind forces as well as in their absence. The present research work is aimed at the reliability-based optimal design of a frame-supported tensile membrane structure subjected to random wind forces. The displacement of the membrane is minimised under this random loading constrained to a stable TMS form and a maximum failure probability of $10^{-4}$ against membrane tearing. A particle swarm optimisation algorithm is used, combined with Latin hypercube sampling, for obtaining the optimum initial prestress values. These algorithms balance the computation heavy dynamic relaxation required for the membrane analysis. The results show that the proposed method can find the required initial prestress for an optimum membrane behaviour under random wind forces, within a manageable level of computation.
\end{abstract}




\section{INTRODUCTION}

Tensile membrane structures (TMS) are lightweight flexible structures that are designed to span long distances with structural efficiency. Their in-plane tensile stresses along with an anticlastic shape provide the necessary resistance to carry external actions of wind or snow. A TMS needs to remain in equilibrium in the presence of heavy wind forces as well as in their absence. The equilibrium, threatened by the inherent flexibility of these structures along with their inability to carry out-of-plane moment and shear, can only be achieved by a proper choice of the initial prestress. In addition, the stresses developed under the wind forces have to be within limits in order to avoid any tearing of the fabric. Due to the highly random nature of wind forces, the designer encounters the challenge of prescribing the right initial prestress values that ensure stability and equilibrium of the structure. This task gets further complicated by the fact that the form-finding of TMS and their structural analysis under static loads are computation intensive.

The present research work is aimed at the reliability-based optimal design of a frame-supported tensile membrane structure. The proposed methodology provides the optimum initial prestress values that give a stable TMS in the presence and absence of random wind forces. The design wind pressure is considered to be following a Type I extreme value distribution. The displacement of the membrane is minimised under this random loading constrained to a stable TMS form and a maximum failure probability of $10^{-4}$ against tearing of the membrane. A particle swarm optimisation (PSO) algorithm is used combined with dynamic relaxation based static analysis of the TMS. The stochastic nature of wind forces is treated using improved probabilistic simulation techniques in order to reduce the computation.

\section{RELIABILITY-BASED OPTIMISATION}

The design process of TMS primarily involves three stages: (a) Form-finding, (b) Static analysis and (c) Patterning [1]. The preliminary design problem of TMS begins with the determination of the initial equilibrium configuration which gives the initial conditions for the subsequent loading analysis under the external wind and/or snow loads that the structure is typically subjected to [2]. In the present work, the TMS is analysed under uncertain wind loads. Dynamic relaxation (DR) with kinetic damping [3] is used as the numerical analysis technique for both form-finding and loading analysis of the membrane structure. The probabilistic distribution of wind load intensity $(W)$ is adopted based on past statistical studies [4], which is also followed in standards like ASCE7. The cumulative distribution function (CDF) of the load intensity is modelled using a Type I distribution with coefficient a of variation, $V_{W}=0.37$ and bias factor, $\lambda_{W}=0.78$ [5]. The nominal value of wind intensity $\left(W_{n}=1 \mathrm{kN} / \mathrm{m}^{2}\right)$ is adopted from a design example for TMS demonstrated in a recent work [6]. The problem of optimisation of the initial prestresses subjected to random wind forces is formulated with an objective to minimise the displacement of the membrane under this random loading and constrained to a stable TMS form and a maximum failure probability of $10^{-4}$ against tearing of the membrane. No wrinkling condition is assumed in the process of form-finding and static analysis which is a reasonable approximation based on past studies [6]. The bounds on initial prestress as a design variable are usually selected by the designer based on the target prestress to be achieved at equilibrium. The objective function and the constraint of the optimisation problem formulated here depends on the design variable (initial prestress). The set of all considered values of the design variable constitutes the 'design space'.

Reliability-based optimisation (RBO) is an efficient methodology to solve structural design 
problems [7]. RBO simultaneously takes into account the optimisation problem while considering the uncertainties associated with structural response. Numerical implementation of RBO in practical engineering problems has always been debated in terms of solution accuracy and intensive computation involved. Particle swarm optimisation (PSO) is a meta-heuristic stochastic optimization technique inspired by social behaviour of animals such as bird flocking or fish schooling. The original idea was to simulate the social behaviour of a swarm of birds [8]. In PSO, a number of entities, called 'particles', adjust their flying speed according to their past movement as well as their partner's flying behaviour. Thus, from the initially generated random particles, PSO probes for the optimum value by updating its particles. Eventually, the swarm as a whole is expected to move close towards the optimum function value.

In the present paper, a decoupled approach of RBDO [9] is adopted to find the optimal solution of the design variable in a reliable domain of search. Dynamic relaxation-based structural analysis of TMS is performed, in combination with probabilistic simulations, to estimate the parameters of interest for the optimisation problem. In order to keep the sensitive areas - that may be susceptible to excessive membrane deformation under wind loading - stable, the objective is to minimise the mean value of the maximum nodal displacement $\left(\mu_{f_{\delta}}\right)$. Also, during the minimisation process, maximum principal stress considering a target structural failure probability of $10^{-4}\left(p_{199.99}\right)$ must be limited to the membrane tearing/yield strength $\left(f_{y}\right)$. Reliability analysis and optimisation are decoupled by transforming the objective function and constraint into approximating functions by fitting regression model. PSO as a stochastic optimisation technique then solves for the optimum value of initial prestress based on the explicit objective (fitness) function. The optima thus obtained is further checked with the implicitly estimated reliability constraints to obtain a global PSO solution. Since form-finding and static analysis of membranes using DR are computation intensive, the number of random particles and random wind intensity samples must be limited while making sure that the level of accuracy in analysis is maintained. To deal with this problem, the randomness in wind intensity is dealt with the efficient variance reduction technique, Latin hypercube sampling (LHS). PSO particles for static analysis with wind intensity samples are chosen initially based on population selection for engineering problems [10]. However, the optimisation is repeated with a larger number of random initial prestress values (PSO particles) and a larger number of probabilistic simulations to check for accuracy.

The RBDO problem is formulated as

$$
\begin{array}{llr}
\underset{p_{i n, w}, p_{i n, f}}{\operatorname{minimise}} & F=\mu_{f_{\delta}} & \\
\text { subject to: } & p_{1_{\max }}<f_{y} & \text { without wind load } \\
& p_{2_{\min }}>0 & \text { without wind load } \\
& p_{1_{99.99}}<f_{y} & \text { with wind load }
\end{array}
$$

where, $p_{1_{\max }}$ and $p_{2_{\min }}$ are the extreme values of maximum and minimum principal stresses after form-finding. $p_{1,99.99}$ is the 99.99 percentile value of the maximum principal membrane stress obtained from the wind load analyses.

The proposed reliability-based optimisation of the initial prestress is outlined in the following steps:

(1) Randomly generate $k$ initial prestress values in the yarn directions.

(2) Perform form-finding using DR for $k$ particles. 
(3) Perform $n$ number of static analyses for each of the $k$ form-found structures under uncertain wind load using DR.

(4) Using MCS with LHS, calculate the reliability-based objective function $\left(\mu_{f_{\delta}}\right)$ and performance measure $\left(p_{1_{99.99}}\right)$ from the static analysis, for each particle.

(5) Obtain an explicit function from the $k$ values of $\mu_{f_{\delta}}$ and $p_{1,99.99}$ by fitting 'response surfaces'.

(6) Optimise using PSO with the formulated RBDO problem given in Eq. 1.

(7) Obtain the optimal design variable (initial prestress) and the corresponding fitness function $\left(\mu_{f_{\delta}}\right)$ value.

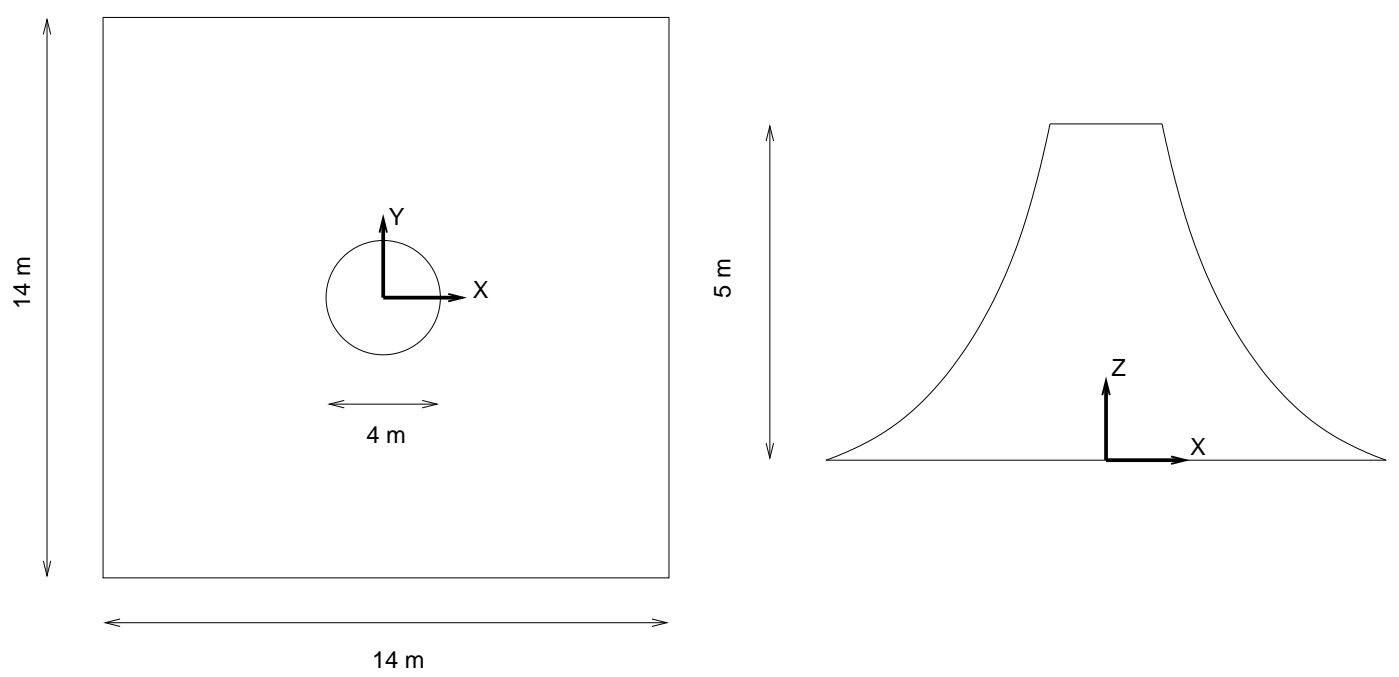

Figure 1: Plan and elevation of the conic tensile membrane structure

\section{A CASE STUDY}

\subsection{Description of the structure}

The tensile membrane structure considered for this case study is a conic structure supported by a square rigid frame at the base. The details of the structure is shown in Figure 1. The following information has been provided for the exercise:

(1) Type and configuration of the boundary supports: Both the square base and the head ring are fixed.

(2) Fabric orientation direction: The principal directions (radial and circumferential) coincide with the fabric yarn (warp and fill) directions.

(3) Fabric initial prestress is applied in warp and fill directions.

(4) Modulus of elasticity, $E=600 \mathrm{kN} / \mathrm{m}$; Poisson's ratio, $\nu=0.4$ and membrane thickness $=$ $1 \mathrm{~mm}$. 
The constant strain triangular (CST) element is used to discretise the membrane structure and the surface topology is defined for the trial shape to start with the analysis. There are 3 translational degrees of freedom per node $\left\{u_{X}, u_{Y}, u_{Z}\right\}$ along the global $\{X, Y, Z\}$ directions. Due to the symmetry about $X$ and $Y$ axes, the structure is analysed for a quarter part only. Symmetric boundary conditions are applied on the edges; with the edge parallel to the $Y$ axis having $u_{X}=0$ and the edge parallel to the $X$ axis having $u_{Y}=0$. Moreover, the base and head rings are fixed with $u_{X}=u_{Y}=u_{Z}=0$.

\subsection{Results and discussion}

In the reliability-based optimisation of initial prestress for the conic TMS under uncertain wind load for the conic structure for $k$ random initial prestress values (PSO particles), DR-based reliability analysis is performed considering 100 random wind intensity samples, as discussed earlier. Figure 2 shows the CDF of maximum principal stress $\left(p_{1_{\max }}\right)$ and resultant displacement $\left(f_{\delta}\right)$ for the random particle with initial prestress $p_{i n, w}=4.629 \mathrm{kN} / \mathrm{m}$ and $p_{i n, f}=3.552 \mathrm{kN} / \mathrm{m}$. For each particle, a generalised pareto (GP) distribution best fits the empirical CDF of $p_{1_{\max }}$, while a generalised extreme value (GEV) distribution best fits the empirical CDF of $f_{\delta}$ based on Kolmogorov-Smirnov goodness-of-fit test for 5\% significance level. Based on the fitted distributions for $p_{1_{\max }}$ and $f_{\delta}$, the values $p_{1_{99.99}}$ and $\mu_{f_{\delta}}$ are calculated for each particle. For the particle under consideration, we get $p_{1_{99.99}}=38.401 \mathrm{kN} / \mathrm{m}$ and $\mu_{f_{\delta}}=3.581 \mathrm{~m}$. Figure 3 shows the values of $p_{199.99}$ and $\mu_{f_{\delta}}$ for this particle plotted against LHS samples of $W$. These plots show that 50 LHS sample are adequate for the reliability analysis considering both the stress and displacement parameters.

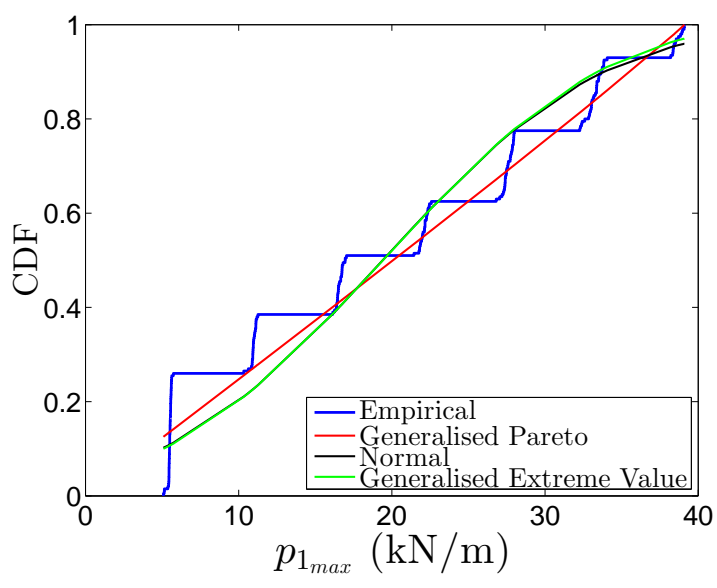

(a)

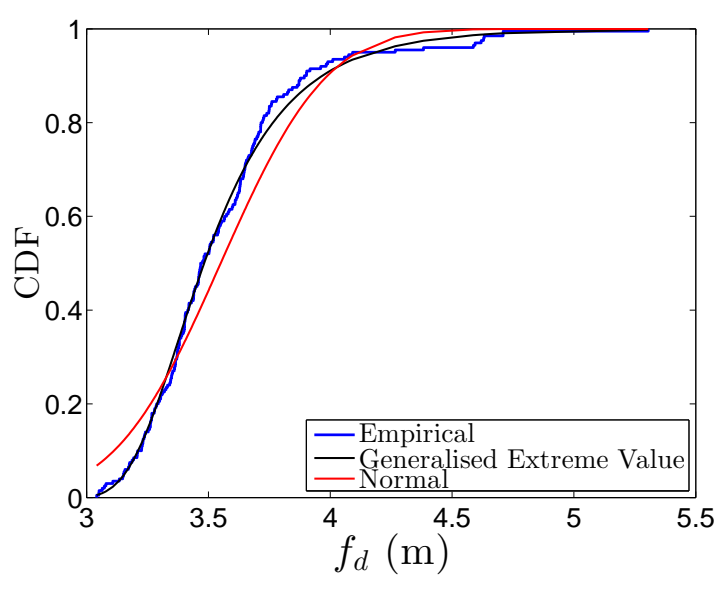

(b)

Figure 2: CDF plots for $p_{1_{\max }}$ and $f_{\delta}$ for a sample PSO particle

As an initial assumption, the value of $k$ is taken as 50 based on the study carried out by Shi et al. [10] on PSO population selection. Thus, dynamic relaxation-based reliability analyses for 50 PSO particles is performed to obtain 50 values of $p_{1_{99.99}}$ and $\mu_{f_{\delta}}$. A response surface is fitted for each of these parameters $\mu_{f_{\delta}}$ and $p_{1_{99.99}}$, in order to obtain the objective function and constraint to be used in the PSO iteration. This process decouples the reliability analysis and the optimisation process, thereby reducing the computation time involved while performing form-finding and static analysis within the optimisation loop. For example, an explicit objective 


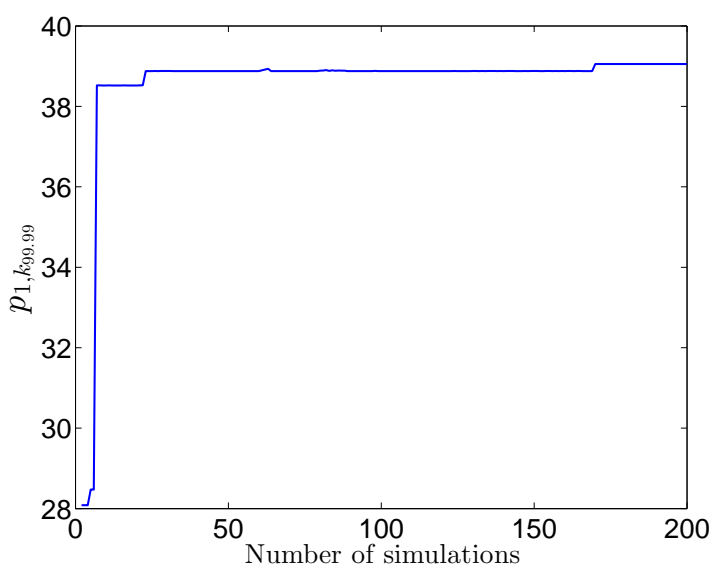

(a)

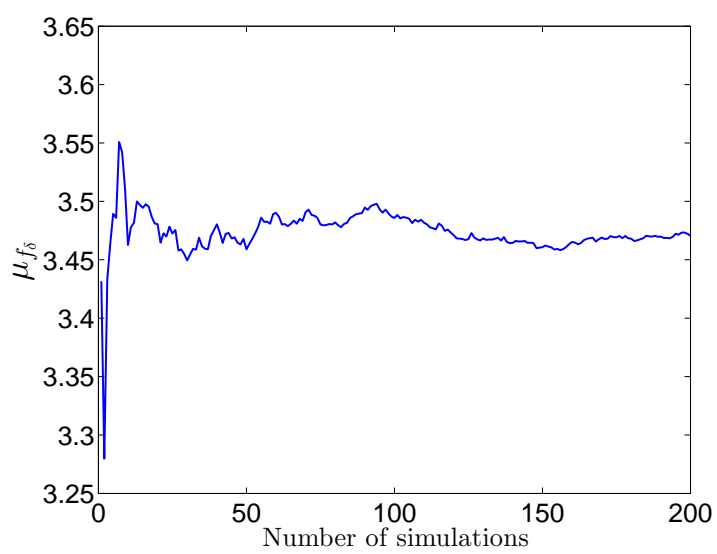

(b)

Figure 3: $p_{1_{99.99}}$ and $\mu_{f_{\delta}}$ plotted against wind intensity samples (LHS)

function for $\mu_{f_{\delta}}$ is obtained by fitting a surface with $p_{i n, w}$ and $p_{i n, f}$ as variables:

$$
\mu_{f_{\delta}}=8.213+0.2151 p_{i n, w}-1.461 p_{i n, f}
$$

which gives a very acceptable value of $R^{2}=0.9731$.

The RBDO problem of Eq. 1 is then solved using PSO to get the optimal $p_{i n, w}$ and $p_{i n, f}$. The fabric tear strength $\left(f_{y}\right)$ is assumed as $40 \mathrm{kN} / \mathrm{m}$. Table 1 provides the RBDO results showing the optimal initial prestress values as the global optimum PSO solution. The optimal initial prestress is thus obtained as $p_{i n, w}=4.953 \mathrm{kN} / \mathrm{m}$ and $p_{i n, w}=3.231 \mathrm{kN} / \mathrm{m}$ with the objective function value, $\mu_{f_{\delta}}=1.657 \mathrm{~m}$. It can been interpreted from this table, that 50 PSO particles gives a converged result in terms of $\mu_{f_{\delta}}$ values. The deflected shape of the conic structure under the design wind intensity of $1 \mathrm{kN} / \mathrm{m}^{2}$ and considering the optimum initial prestress values is shown in Figure 4. From this deflected shape, the maximum nodal displacement, $f_{\delta}$ is found to be $2.753 \mathrm{~m}$, with a maximum value of principal stress $p_{1_{\max }}=27.36 \mathrm{kN} / \mathrm{m}$.

Table 1: RBDO results

\begin{tabular}{llll}
\hline \hline Particles & $p_{i n, w}(\mathrm{kN} / \mathrm{m})$ & $p_{i n, f}(\mathrm{kN} / \mathrm{m})$ & $\mu_{f_{\delta}}(\mathrm{m})$ \\
\hline 50 & 4.953 & 3.231 & 1.657 \\
100 & 4.981 & 3.269 & 1.648 \\
\hline \hline
\end{tabular}

\section{CONCLUSIONS}

In this paper, an optimisation under uncertainty problem is presented in the conception of a tensile membrane structure. Optimisation of the TMS in terms of its strength and stability under uncertain wind load is achieved by varying the initial prestress. The effectiveness of the stochastic particle swarm optimisation algorithm, along with the stratified Latin hypercube sampling, is studied in this work, which employs the dynamic relaxation algorithm with kinetic damping for structural analysis of the membrane structure. The proposed framework provides a solution to this RBDO problem without compensating the accuracy level achieved for the computation time involved. The proposed methodology is demonstrated for a conic TMS with a square rigid base. The efficiency of this RBDO approach is reflected by the minimisation 


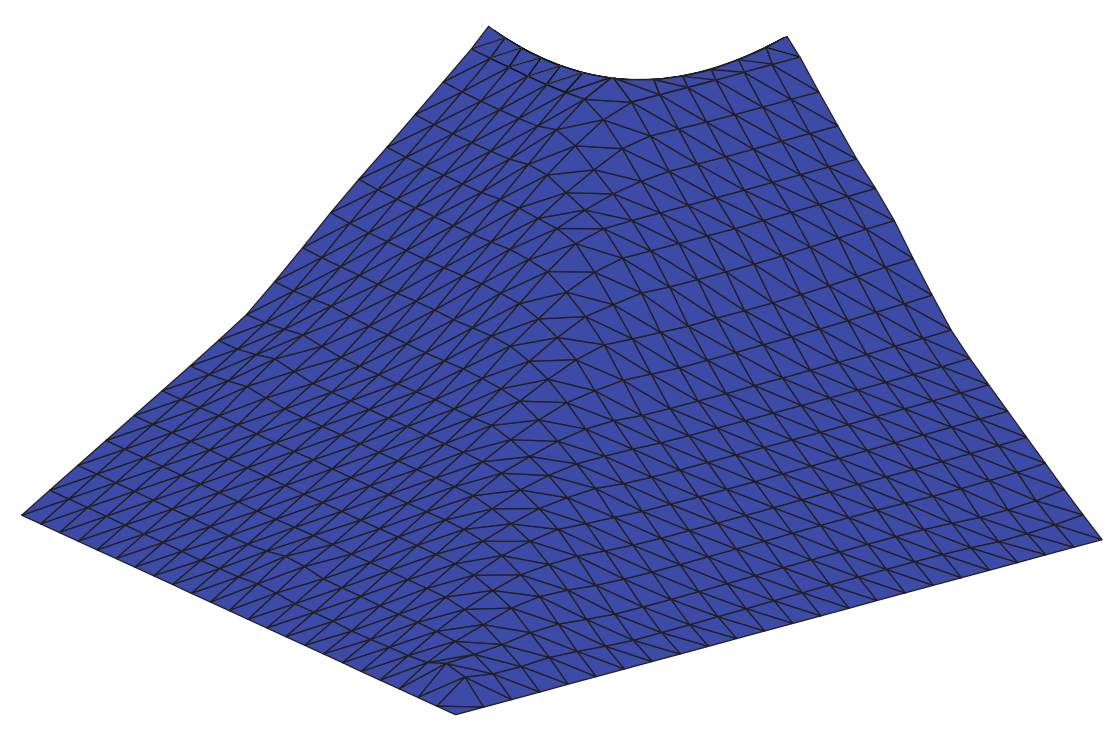

Figure 4: Deflected shape of the structure (quarter part) under the design wind load

of deformations and by limiting the probability of membrane tearing to a specific probability under uncertain wind loads.

Future direction of work may include trials with advanced probabilistic/optimisation techniques, for example surrogate-based modelling, with due emphasis on accuracy and computation time. Furthermore, the design can be further improved by an efficient multi-objective RBDO formulation for different loading conditions. The findings from this study provides an insight to TMS designers to adopt a probabilistic optimisation approach for a better design of tensile membrane structures that would satisfy strength and stability requirements.

\section{References}

[1] W. J. Lewis. Modeling of fabric structures and associated design issues. Journal of Architectural Engineering, ASCE, 19(2):81-88, 2013.

[2] C. G. Huntington. Tensile Fabric Structures: Design, Analysis and Construction. American Society of Civil Engineers, 2013.

[3] W. J. Lewis. Tension Structures Form and Behaviour. Thomas Telford Publishing, London, 2003.

[4] B. Ellingwood. Wind and snow load statistics for probabilistic design. Journal of the Structural Division, ASCE, 107(ST7):1345-1350, 1981.

[5] B. R. Ellingwood and P. B. Tekie. Wind load statistics for probability-based structural design. Journal of Structural Engineering, ASCE, 125(4):453-463, 1999.

[6] P. D. Gosling, B. N. Bridgens, A. Albrecht, H. Alpermann, A. Angeleri, M. Barnes, N. Bartle, R. Canobbio, F. Dieringer, S. Gellin, W. J. Lewis, N. Mageau, R. Mahadevan, J. . Marion, P. Marsden, E. Milligan, Y. P. Phang, K. Sahlin, B. Stimpfle, O. Suire, and J. Uhlemann. Analysis and design of membrane structures: Results of a round robin exercise. Engineering Structures, 48:313-328, 2013.

[7] W. Li and L. Yang. An effective optimization procedure based on structural reliability. Computers and Structures, 52(5):1061-1071, 1994. 
[8] J Kennedy and R Eberhart. Particle swarm optimization. In Proceedings of the IEEE International Conference on Neural Networks, volume 4, pages 1942-1948, 1995.

[9] T. Zou and S. Mahadevan. A direct decoupling approach for efficient reliability-based design optimization. Structural and Multidisciplinary Optimization, 31(3):190-200, 2006.

[10] R. C. Eberhart and Y. Shi. Particle swarm optimization: Developments, applications and resources. In Proceedings of the IEEE Conference on Evolutionary Computation, ICEC, volume 1, pages 81-86, 2001. 